\title{
Standardisation de la classification morpholo- gique des spermatozoïdes humains selon la méthode de David modifiée
}

\author{
J. AUGER, F. EUSTACHE
}

En hommage au Professeur G. David

Service de Biologie de la Reproduction / CECOS, Hôpital Cochin, Paris

\section{RÉSUMÉ}

Il est maintenant bien établi que le pourcentage de spermatozoïdes normaux et de certaines anomalies spécifiques des spermatozoïdes a une valeur pronostique in vivo et in vitro. Aussi, le spermocytogramme représente un temps indispensable de l'analyse du sperme humain. Malheureusement, cette analyse, simple à première vue, présente de réelles difficultés avec pour conséquence une fiabilité très relative des résultats d'un laboratoire à l'autre. Aussi, les procédures employées, de la réalisation des frottis au rendu des résultats, doivent faire l'objet d'une standardisation. La lecture des lames colorées de préférence au Shorr est faite à l'objectif x100 à immersion. La méthode de David modifiée pour le classement des anomalies morphologiques des spermatozoïdes humains a été adoptée en France par la grande majorité des laboratoires publics et privés. Elle permet le classement de $1^{\circ}$ ) sept anomalies de la tête : têtes allongées, amincies, microcéphales, macrocéphales, multiples, présentant un acrosome anormal ou absent, présentant une base (région post-acrosomique) anormale, $2^{\circ}$ ) trois anomalies de la pièce intermédiaire : reste cytoplasmique, grêle, angulée et $3^{\circ}$ ) cinq anomalies de la pièce principale : absente, écourtée, de calibre irrégulier, enroulée et multiple. La grande originalité de la méthode est de recenser l'ensemble des anomalies associées par spermatozoïde anormal grâce à un système à entrées multiples. Comme il n'y a aucune raison objective de considérer plus une anomalie qu'une autre, il est impératif de décrire toutes les anomalies observées pour chaque spermatozoïde anormal. L'index d'anomalies multiples ou IAM, application directe de ce système n'est autre que le rapport du nombre total d'anomalies recensées au nombre total de spermatozoïdes anormaux. L'IAM, indicateur du nombre moyen d'anomalies associées par spermatozoïde anormal, présente une valeur pronostique de la fertilité et l'OMS recommande son utilisation. La présente mise au point s'inscrivant dans la démarche nécessaire d'assurance de qualité en biologie de la reproduction est focalisée sur une redéfinition précise des anomalies, les justifications ultrastructurales et fonctionnelles et la pratique du classement des spermatozoïdes humains typiques et atypiques selon la méthode de David modifiée.

Mots clés : Analyse morphologique, spermatozoïde humain, spermocytogramme, standardisation, assurance de qualité, index d'anomalies multiples.

Correspondance : J. Auger, Service de Biologie de la Reproduction/CECOS, Hôpital Cochin, 123, Bd de Port Royal, 75014 Paris

Communication aux 3èmes Journées Nationales Assurance de Qualité en Biologie de la Reproduction, 26 \& 27 Octobre 2000, Nancy 


\section{INTRODUCTION}

Le spermatozoïde est une cellule hautement différenciée dont l'organisation structurale est spécialement et exclusivement adaptée à la rencontre et à la fusion avec l'ovocyte. Cette organisation structurale originale est le résultat de processus morphogenétiques complexes se déroulant pendant la spermiogenèse, étape fondamentale de la spermatogenèse. Chez l'homme, des spermatozoïdes de morphologie typique et des spermatozoïdes présentant des anomalies morphologiques diverses sont produits, ces derniers étant généralement plus représentés si l'on se réfère au seuil de normalité de l'OMS se situant à $30 \%$ et plus de spermatozoïdes morphologiquement normaux [16]. Les spermatozoïdes morphologiquement anormaux ont un potentiel fécondant réduit (voire aboli dans le cas des spermatozoïdes sans acrosome) et ce d'autant plus qu'ils cumulent des anomalies.

Il est maintenant bien établi que le pourcentage de spermatozoïdes normaux a une valeur pronostique in vivo $[3,7,11,18]$ et in vitro $[10$, 12, 13]. Par ailleurs, il a été rapporté que la proportion de certaines anomalies spécifiques des spermatozoïdes et le nombre moyen d'anomalies par spermatozoïde atypique, l'index d'anomalies multiples, IAM $[11,16]$, avaient un intérêt pronostique in vivo ou in vitro [11, 10]. Enfin, la vulnérabilité du testicule humain à de nombreux facteurs physiques et chimiques [17] ou à des facteurs plus complexes tels que le stress [9], a suggéré que l'analyse des anomalies morphologiques des spermatozoïdes, pouvait constituer un indicateur utile des facteurs du macro-environnement de l'homme pouvant moduler ou endommager la spermatogenèse $[14,20]$.

Ainsi, le spermocytogramme, appellation usuelle pour l'analyse morphologique des spermatozoïdes humains - comprenant l'évaluation du pourcentage de spermatozoïdes morphologiquement normaux et la détermination de l'incidence des diverses anomalies morphologiques - représente plus que jamais un temps indispensable de l'analyse du sperme humain. Malheureusement, cette analyse, simple à première vue, présente de réelles difficultés avec pour conséquence une fiabilité relative des résultats d'un laboratoire à l'autre.

\section{Ces difficultés tiennent :}

1. à l'utilisation de systèmes de classification multiples : une dizaine de systèmes de classification des anomalies morphologiques des spermatozoïdes humains ont été proposés depuis la fin des années 50, sans consensus encore actuellement sur une méthode de classification universelle; cette situation est bien illustrée dans le manuel de l'OMS : "Many abnormal spermatozoa have multiple defects... Morphological assessment should be multiparametric for each sperm, tallying each defect separately" et, un peu plus bas : "It is considered unnecessary routinely to distinguish between all the variations in size-shape and other defects or between the various tail defects" [19],

2. à l'absence de définition précise et détaillée dans la plupart des systèmes de classification et à l'absence de règle pour le classement des spermatozoïdes présentant plus d'une anomalie morphologique,

3. au caractère subjectif de l'analyse microscopique de la morphologie des spermatozoïdes humains qui dépend principalement des mécanismes de la vision et de son intégration au niveau cérébral.

Lors de l'analyse des anomalies morphologiques des spermatozoïdes humains, l'observateur doit estimer les tailles respectives des différents spermatozoïdes ou de leurs constituants (têtes des spermatozoïdes trop petites ou trop grandes, longueur du flagelle, par exemple) et reconnaître des formes (spermatozoïdes à flagelles multiples, à flagelle enroulé ou sans flagelle, par exemple). Le couple oeilcerveau est très efficace pour la reconnaissance des formes, mais il est peu performant et beaucoup moins efficace que la vision assistée par ordinateur pour l'évaluation de tailles respectives d'éléments microscopiques. C'est la raison pour laquelle des systèmes de classification comme la classification de Tygerberg [12] basée uniquement sur une évaluation très stricte des seuls spermatozoïdes normaux (les spermatozoïdes "top model" !) peuvent apparaître à première vue d'une utilisation plus aisée bien qu'une meilleure reproductibilité ou un plus grand intérêt clinique avec cette 
méthode n'ait encore été démontré. C'est aussi la raison pour laquelle des méthodes d'analyse semi-automatisées à l'aide de logiciels spécifiques de vision par ordinateur ont été proposées pour tenter de pallier à la subjectivité de l'analyse visuelle pour l'analyse de la morphologie spermatique [4, 15]. Malheureusement, dans leur état actuel de développement, ces méthodes ne sont pas recommandées [8].

L'étape analytique de reconnaissance visuelle et de classification des spermatozoïdes humains normaux et anormaux doit donc impérativement faire l'objet d'une standardisation. Les phases importantes de la standardisation sont d'une part, l'optimisation de la préparation et de l'observation microscopique, afin d'avoir les meilleures conditions possibles de contraste des cellules, et d'autre part, la définition précise de chaque atypie afin que les critères de décision pour la classification soient les mêmes d'un observateur à l'autre. Le présent article est centré sur la définition de la morphologie normale et des atypies spermatiques et sur la pratique de la classification des spermatozoïdes normaux et anormaux dans le cadre de l'utilisation de la méthode de David modifiée.

\section{PRÉPARATION DES FROTTIS, COLORATION, GROSSISSEMENT, MÉTHODE POUR LA LECTURE}

Dans notre laboratoire, les frottis sont réalisés selon l'une des techniques de confection proposées dans le manuel de l'OMS pour l'analyse du sperme [16]. La technique consiste à déposer 10 ul de sperme bien homogénéisé à l'extrémité d'une lame et à étaler cette goutte en s'aidant d'une autre lame inclinée à $45^{\circ}$ par rapport à la première. On obtient dans ces conditions un frottis très peu épais limitant de possibles artefacts de coloration du fond de la préparation. Ainsi, un contraste optimal des cellules après coloration est obtenu. Les frottis une fois séchés à l'air sont fixés dans un mélange 3/4 éthanol, $1 / 4$ acide acétique pendant 1 minute.

L'OMS dans son manuel pour l'analyse du sperme [16] ne s'est pas prononcée sur la technique de coloration optimale pour les spermatozoïdes humains. Elle indique les colorations de Giemsa, de Papanicolaou modifiée pour les spermatozoïdes, de Bryan-Leishman ou encore la coloration de Shorr. Une enquête du groupe de travail national sur l'assurance de qualité en biologie de la reproduction auprès de 214 laboratoires français avait montré que plus de cinq techniques différentes étaient utilisées pour la coloration des spermatozoïdes ! [5]. Il serait souhaitable qu'une technique unique soit retenue afin d'améliorer la standardisation d'un laboratoire à un autre. Dans notre laboratoire, nous utilisons la coloration de Shorr qui est simple à réaliser et donne de bons résultats pour une évaluation en routine de la morphologie des spermatozoïdes, en particulier, elle semble supérieure à la méthode de Papanicolaou modifiée pour la reconnaissance des pièces intermédiaires et des flagelles. La coloration est faite à l'aide d'un automate de coloration Sakura pour 50 à 100 lames, ce qui permet une homogénéité d'une lame à l'autre. Les lames sont ensuite montées à l'Eukitt.

La lecture des lames colorées est faite à l'objectif x100 à immersion (soit un grossissement final de x1000 avec des oculaires de x10). L'un des oculaires est muni d'un réticule gradué dont l'acquisition est très recommandée pour prendre une décision de classification à chaque fois que l'on hésitera sur un critère de taille (tête amincie par rapport à tête normale, par exemple). La lecture doit être faite en queue de frottis sur des champs microscopiques jointifs, avec un balayage en "méandre" de la lame et des champs. Si l'échantillon est très concentré, on ne doit pas lire sur un seul champ.

Les têtes des spermatozoïdes plus ou moins normaux présentent une face et un profil : elles se couchent donc lors de la réalisation du frottis, se présentant ainsi de face pour l'observation. Cependant, dans certains spermes avec des anomalies de la viscosité, des spermatozoïdes en nombre plus ou moins important peuvent se présenter de profil. L'aspect de profil est assez caractéristique, en "flamme de bougie" et la texture est homogène sans distinction d'une région acrosomique. Ces spermatozoïdes doivent être reconnus comme tels et ne pas être confondus avec des spermatozoïdes avec une tête amincie (voir plus loin) et il ne faut pas les classer car il est impossible de faire une description morphologique à partir d'un profil. 


\section{MÉTHODE DE CLASSIFICATION DE DAVID : PRINCIPE, DÉFINITIONS ET EXEMPLES}

\section{Principe}

La méthode de David pour le classement des anomalies morphologiques des spermatozoïdes humains a été proposée en 1972 lors d'un colloque sur la fécondité et la stérilité du mâle et publiée en 1975 [6]. La classification initiale comportait 13 types d'anomalies, sept pour la tête et six pour le flagelle. L'originalité de la méthode de classification repose sur la prise en compte de toutes les anomalies observées grâce à un système de classification à entrées multiples : un spermatozoïde porteur de plusieurs anomalies est défini par l'ensemble de ces anomalies c'est-à-dire qu'il figure plusieurs fois dans la grille de classement, à chacune des anomalies concernées.

Depuis les années soixante-dix, de nombreux laboratoires français, publics et privés ont adopté cette classification et, à l'initiative de plusieurs biologistes l'utilisant très régulièrement, elle a été modifiée au début des années quatre-vingt-dix par une redéfinition des catégories d'anomalies et l'adjonction de deux nouvelles catégories d'anomalies. Cette modification correspondait à un souci accru des biologistes de la reproduction de voir figurer dans la grille toute atypie morphologique reflétant un possible défaut ultrastructural susceptible d'avoir un retentissement fonctionnel. Ainsi la catégorie "têtes irrégulières" a-t-elle été scindée en deux catégories distinguant d'une part les atypies de la région acrosomique et, d'autre part, les atypies de la région post-acrosomique (anomalies de forme et/ou de contour et/ou de texture, dans les deux cas) pouvant toutes deux être à l'origine de perturbations de l'interaction gamétique.

La classification de David modifiée recense en dehors des spermatozoïdes morphologiquement normaux :

- 7 anomalies de la tête : têtes allongées, amincies, microcéphales, macrocéphales, multiples, présentant un acrosome anormal ou absent, présentant une base (région postacrosomique) anormale
- 3 anomalies de la pièce intermédiaire (PI) : reste cytoplasmique, PI grêle, PI angulée et,

- 5 anomalies de la pièce principale : absente, écourtée, de calibre irrégulier, enroulée et multiple

Compte tenu de l'inhomogénéité du sperme humain et de la faible fréquence de certaines anomalies, 100 spermatozoïdes au minimum doivent être classés pour fournir une évaluation correcte du pourcentage de spermatozoïdes typiques et du profil des différentes atypies. Cette recommandation est cependant parfois impossible à suivre lorsque la concentration de spermatozoïdes est très faible. Dans ces cas, la classification peut être faite à partir de 50 spermatozoïdes, mais le résultat pour les spermatozoïdes normaux et anormaux ne doit pas être rendu en pourcentage et la conclusion doit tenir compte de la fiabilité diminuée des fréquences des anomalies retrouvées, notamment lorsqu'il s'agit d'anomalies rares.

Les flagelles isolés ou les spermatozoïdes en lyse ne sont pas classés dans la grille de lecture modifiée, mais leur fréquence est évaluée parallèlement au compte des spermatozoïdes normaux et anomaux. Il en est de même pour la fréquence des cellules de la lignée blanche, des autres cellules (cellules du tractus urogénital) et des fragments cellulaires.

\section{INDEX D'ANOMALIES MULTIPLES, IAM}

Lors de la refonte de la grille de lecture, il a été décidé de faire figurer systématiquement l'index d'anomalies multiple ou IAM, proposé en 1988 [11], à côté du pourcentage de spermatozoïdes normaux et du détail des anomalies recensées. Cet index est en fait une application directe du système original à entrées multiples de la méthode : l'IAM n'est autre que le rapport du nombre total d'anomalies recensées au nombre total de spermatozoïdes anormaux. L'IAM est donc un indicateur du nombre moyen d'anomalies associées par spermatozoïde anormal.

\section{DÉfinitions}

\section{a) Spermatozoïdes normaux (Figure 1)}

L'étude des spermatozoïdes récupérés après migration dans le mucus cervical pré-ovulatoi- 
re a aidé à définir les caractéristiques du spermatozoïde normal. En effet, les spermatozoïdes présentant des anomalies de structure diverses sont retenus par le filtre naturel que constitue le mucus cervical pré-ovualtoire.

Définition : La tête a un contour très régulier ovalaire avec un grand axe mesurant $5 \mu \mathrm{m}$ et un petit axe mesurant $3 \mu \mathrm{m}$ (rapport grand axe / petit axe $=1,66$ ). La longueur et / ou la largeur de la tête peuvent être légèrement diminuées sans que celle-ci soit pour autant considérée comme anormale. Le rapport possible grand axe/petit axe peut done fluctuer entre 1,33 et 2. L'acrosome doit couvrir 40 à $70 \%$ de la surface de la tête, avoir un contour régulier et une texture homogène. La pièce intermédiaire normale peu visible en microscopie conventionnelle mesure de 1,5 à 2 fois la longueur de la tête, a un diamètre de 0,6 à $0,8 \mu \mathrm{m}$, son grand axe est dans le prolongement $d u$ grand axe de la tête, présente un contour régulier, une texture homogène et un reste cytoplasmique de taille minime à son niveau n'est pas considéré comme anormal. Enfin, la pièce principale, c'est-à-dire le reste du flagelle, mesure environ $45 \mathrm{\mu m}$ (soit environ 10 fois la longueur de la tête), a un diamètre de l'ordre de 0,4 à $0,5 \mu \mathrm{m}$, est développée avec un contour régulier et un aspect homogène.

Il faut noter que l'ensemble du flagelle jusqu'à la pièce terminale ne se situe pas toujours dans le même plan focal : aussi faut-il jouer en permanence sur la vis micrométrique pour bien décrire le flagelle.

\section{b) Spermatozoïdes anormaux}

Les catégories d'anomalies définies dans la classification en microscopie optique découlent des observations faites en microscopie électronique à transmission [2] et des possibles implications fonctionnelles. Pour chacune d'entre elles, une définition précise est donnée avec l'illustration correspondante et le lien entre l'aspect en microscopie conventionnelle et le substratum ultrastructural est brièvement exposé.

En pratique, à chaque fois que possible, on utilise les spermatozoïdes normaux comme "comparateur visuel" pour classer les différentes atypies et notamment les atypies de taille de la tête ou du flagelle. Ce travail de comparaison est facilité lorsque les spermatozoïdes jugés normaux sont suffisamment nombreux pour que des spermatozoïdes normaux et anormaux se situent dans le même champ microscopique. Sinon il faut balayer la préparation à la recherche de spermatozoïdes typiques à chaque fois que l'on a un doute sur la taille de la tête ou du flagelle du spermatozoïde en cours d'observation. L'utilisation du réticule gradué est recommandée pour l'aide à la décision dans ces situations et encore plus lorsque aucun spermatozoïde de la préparation semble normal : dans ce cas, on doit se référer aux critères de taille du spermatozoïde normal (voir plus haut) en s'aidant du micromètre oculaire.

TAILLE DE LA TÊTE ANORMALE : têtes allongées, amincies, microcéphalie et macrocéphalie (Figure 2)

Toutes ces anomalies morphologiques sont en rapport avec un défaut de la morphogenèse de la tête et/ou du noyau. Il existe une assez bonne concordance entre l'aspect des anomalies de forme observées en microscopie optique et en microscopie électronique à transmission [2]. Pour ces spermatozoïdes, Ies anomalies du matériel nucléaire mises en évidence par cytométrie d'image en microscopie électronique à transmission sont beaucoup plus fréquentes que parmi les spermatozoïdes normaux [1]. Il en est de même lorsqu'on utilise des colorations spécifiques du noyau, bleu d'aniline pour l'évaluation de la maturité nucléaire, acridine orange pour l'évaluation de l'intégrité de l'ADN, Feulgen pour l'évaluation de la ploïdie (les spermatozoïdes macrocéphales sont le plus souvent polyploïdes).

Pour toutes ces anomalies, le réticule gradué ou micromètre oculaire sera utilisé à chaque fois que l'on a un doute sur la taille.

\section{TÊTE ALLONGÉE}

Définition : Le grand axe est plus long que la normale et le petit axe présente une longueur normale.

\section{Tête AMINCIE}

Définition : Le petit axe a une longueur plus petite que la normale et le grand axe présente une longueur normale. 


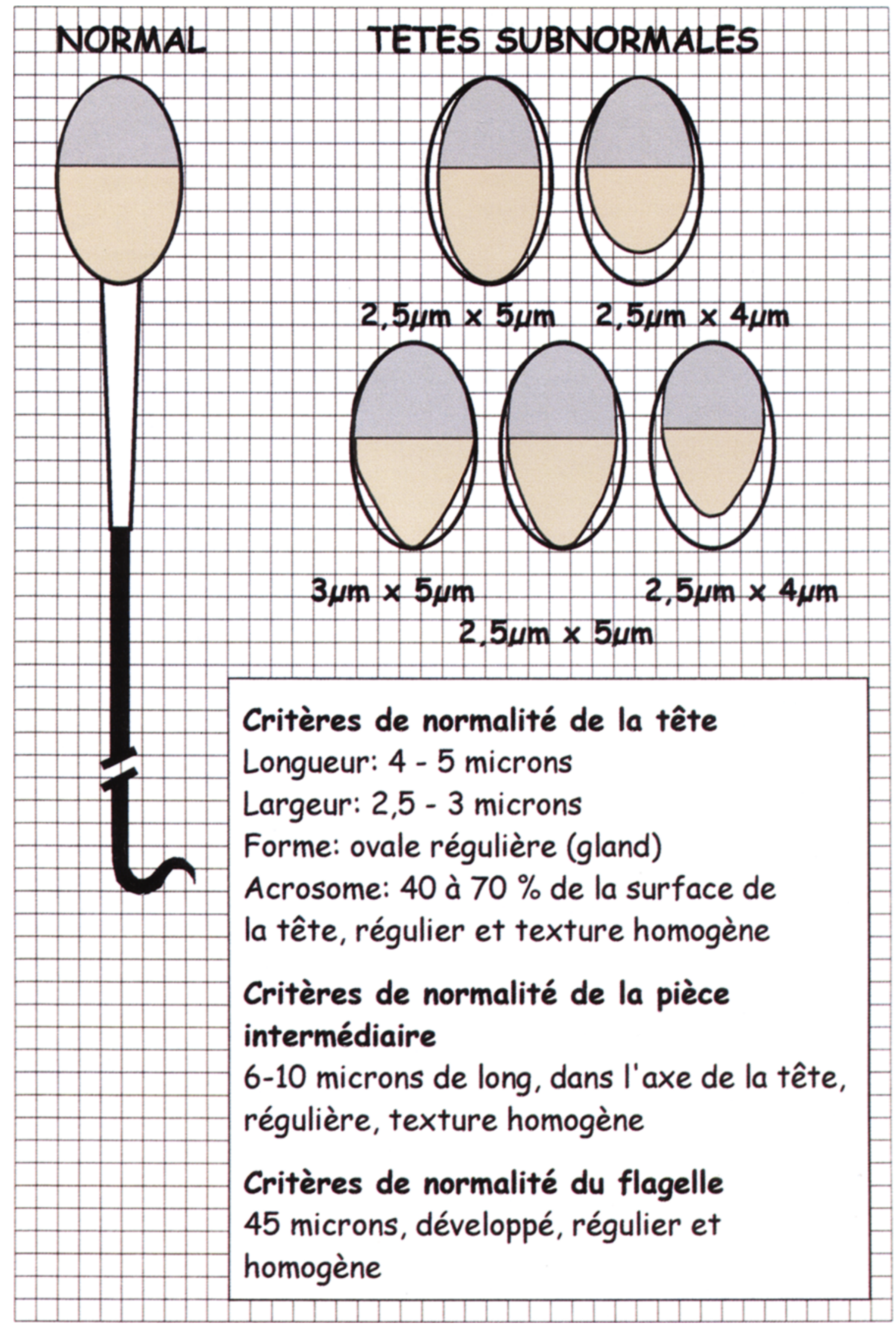

Figure 1 : Illustrations du spermatozoüde humain normal ou subnormal, considéré comme normal, et rappel des définitions. 


\section{MiCROCÉPHALE}

Définition : Le grand axe et le petit axe ont des longueurs plus petites que la normale.

Dans cette catégorie entrent les têtes rondes le plus souvent dépourvues d'un acrosome, mais il existe d'autres aspects de spermatozoïdes microcéphales avec un acrosome plus ou moins normal.

\section{MACROCÉPHALE}

Définition : Le grand axe et le petit axe sont plus grands que la normale.

TÊTES MULTIPles (Figure 3)

Définition : Il y a plus d'une tête par spermatozoïde. Elles peuvent être accolées et occuper une surface totale similaire à celle d'une seule tête ou bien être parfaitement dissociées.

En microscopie électronique à transmission, on constate que ces anomalies sont dues à la persistance de ponts cytoplasmiques plus ou moins importants entre des cellules germinales d'une même onde spermatogenétique qui font que les noyaux demeurent contigus (formes binuclées) ou que des têtes bien individualisées restent associées (formes bicéphales) avec parfois un début de dédoublement de la pièce intermédiaire. On peut observer des anomalies multiples concernant trois noyaux ou têtes et plus.

\section{ANomalies de L'ACRosome (Figure 3)}

Définition : On classe dans cette catégorie toute anomalie de taille, de contour ou de texture de la région acrosomique ainsi que l'absence d'acrosome.

La surface de l'acrosome est inférieure à $40 \%$ ou supérieure à $70 \%$ de la surface totale de la tête (cette évaluation n'est pas toujours aisée car il n'existe pas toujours une limite nette entre régions acrosomique et post-acrosomique: en cas de doute, s'aider de la comparaison avec les spermatozoïdes normaux et utiliser si nécessaire le réticule gradué en le plaçant le long du grand axe et en comparant les rapports de longueur de l'acrosome et de la tête) et /ou, le contour de l'acrosome est irrégulier (il peut y avoir une ou plusieurs encoches sur le contour externe ou bien la limite interne n'est pas rectiligne et/ou non perpendiculaire au grand axe) et/ou, la texture de l'acrosome est inhomogène (avec des aspects de vacuoles irrégulières ; attention des vacuoles bien circulaires avec un halo de colorant correspondent le plus souvent à des artefacts de préparation et ne doivent pas être notées comme de vraies vacuoles) ou, l'acrosome est absent

La microscopie électronique à transmission révèle de très nombreuses anomalies de forme, de taille et de positionnement de l'acrosome par rapport au noyau, qui peuvent affecter l'interaction gamétique.

ANOMALIES DE LA BASE DE LA TÊTE OU RÉGION POST-ACROSOMIQUE (Figure 3)

Définition : Toutes les anomalies de contour et de texture de la région post-acrosomique; le contour doit normalement correspondre à une courbe bien régulière et il faut donc retenir que toute irrégularité dans cette partie de la tête doit être recensée.

En principe, cette partie de la tête est assez uniformément colorée car correspondant à la projection de la partie épaisse et compacte du noyau : les anomalies de texture sont donc assez rares. Par contre, les anomalies de contour sont fréquentes. Il est impossible de toutes les répertorier et la Figure 3 donne des exemples des formes les plus courantes : base rétrécie de chaque côté : "flamme de bougie retournée", base rétrécie d'un seul côté : "virgule", striction de chaque côté : "battant de cloche" (le plus souvent associé à une tête allongée), aspect de "cou carré", "collerette équatoriale" (généralement plus colorée), base carrée : "esquimau".

Toutes ces anomalies morphologiques de la base présentent une assez bonne concordance avec ce qui est observé en microscopie électronique à transmission [2, 1]. Ces anomalies semblent correspondre à un défaut de la morphogenèse de la tête et/ou du noyau dans sa partie distale. En microscopie électronique à transmission, les anomalies de contour de la base sont le plus souvent associées à des anomalies des structures adjacentes et notamment de la cape post-acrosomique qui joue un rôle prédominant dans l'interaction du spermatozoïde avec l'ovocyte. 

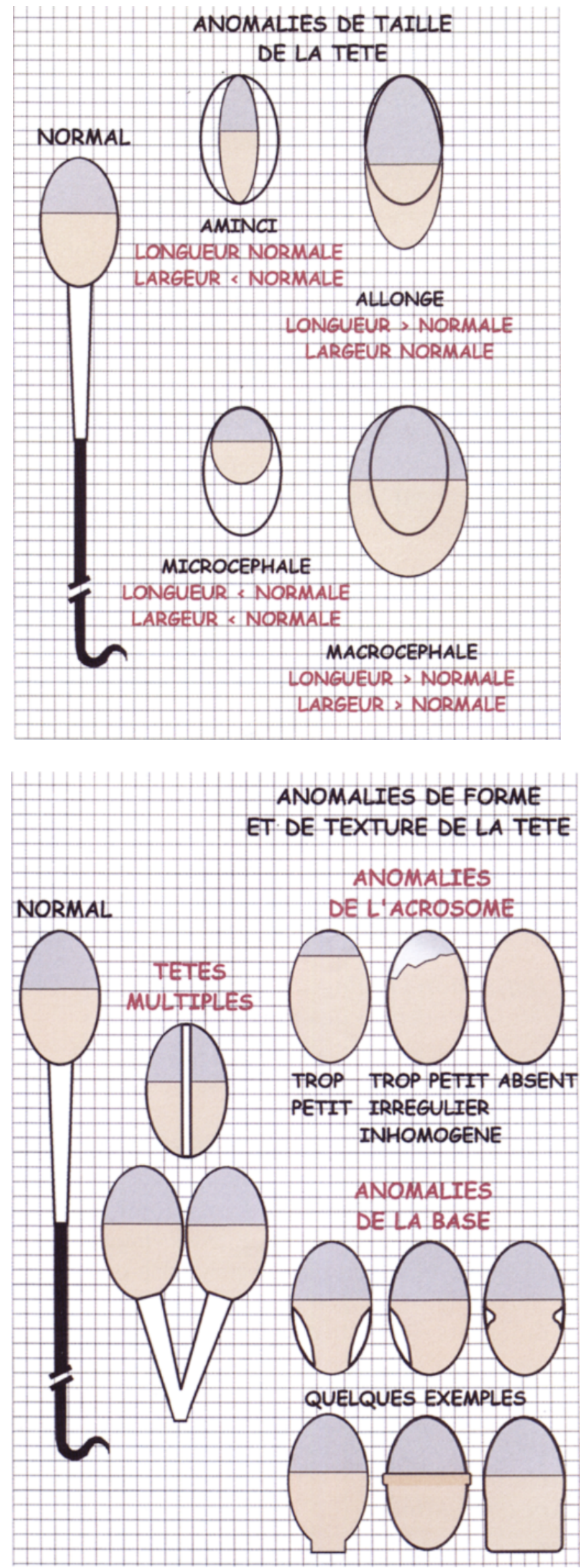

Figure 2 : Illustrations des anomalies de taille de la tête des spermatozoüdes humains.
Figure 3 : Illustrations des anomalies de forme et de texture de la tête des spermatozoüdes humains. 


\section{RESTE CYTOPLASMiQUE (Figure 4)}

DÉFINITION : le reste cytoplasmique est considéré comme une anomalie s'il a une surface supérieure au tiers de la surface d'une tête normale. Il se situe le plus souvent à la jonction de la tête et de la pièce intermédiaire et c'est pourquoi il est classé comme anomalie de la pièce intermédiaire mais il peut également entourer l'ensemble de la cellule (souvent dans le cas de spermatozoïdes enroulés) ou englober seulement la tête.

Normalement, l'essentiel du cytoplasme des spermatozoïdes est éliminé lors de l'excrétion des spermatozoïdes testiculaires dans la lumière des tubes séminifères. Un reste cytoplasmique de taille importante est un signe d'immaturité cellulaire : soit les spermatozoïdes se séparent prématurément de l'épithélium germinal soit le processus de reprise du cytoplasme par les cellules de Sertoli lors de l'excrétion des spermatozoïdes dans le tube séminifère (spermiation) fait défaut. Tous ces restes cytoplasmiques sont clairement mis en évidence en microscopie électronique à transmission.

\section{Pièce inTermédiame GRêLe (Figure 4)}

Définition : le diamètre de la pièce intermédiaire est égal ou inférieur au diamètre de la pièce principale dans sa partie initiale.

Cette anomalie correspond à une gaine mitochondriale qui ne s'est pas constituée.

Angulations du FlaGelle (Figure 4)

Définition : l'axe de la pièce intermédiaire et l'axe de la tête ou l'axe de la pièce principale forment un angle net ou encore le flagelle n'est pas implanté dans l'axe de la tête.

Les angulations sont bien visibles en microscopie électronique. Elles se présentent sous la forme d'une plicature à la jonction de la pièce intermédiaire et de la pièce principale ou plus loin sur le flagelle et régulièrement les structures axonémales et périaxonémales en regard sont désorganisées. Certains aspects d'angulation souvent aiguë ou de désaxement se situent à la jonction de la tête et de la pièce intermédiaire. Ils correspondent à des défauts ultrastructuraux d'un autre type car intéressant les organites de la région du cou. Par commodité, ils sont classés avec les angulations flagellaires au niveau de la pièce intermédiaire.

Flagelle ABSENT (Figure 5)

Définition : les têtes isolées sont comptées dans cette catégorie

La pathologie ultrastructurale intéresse les colonnes striées qui sont absentes et/ou la pièce connective qui est rudimentaire.

Flagelle COURT (Figure 5)

Définition : le flagelle est significativement écourté (<5 fois la longueur de la tête).

La microscopie électronique à transmission indique parfois que le flagelle est court du fait d'une brièveté de la pièce intermédiaire mais, le plus souvent, on observe des flagelles courts et épaissis avec une prolifération des éléments composant la gaine fibreuse. Ces flagelles ne sont pas fonctionnels.

Flagelle irRégulier (Figure 5)

Définition : le diamètre de la pièce principale est variable, présentant des rétrécissements ou des élargissements.

En microscopie électronique à transmission, cette anomalie correspond à des désorganisations étagées de l'axonème et/ou du périaxonème.

\section{Flagelle ENRoulé (Figure 5)}

Définition : Le flagelle est enroulé autour de la tête ou en dehors de la tête.

En microscopie électronique à transmission, on observe des coupes transversales multiples de flagelles correspondant à des niveaux différents au sein d'un même reste cytoplasmique, parfois des incidences longitudinales plus rares montrant nettement un enroulement $d u$ flagelle autour de la tête. Sur les coupes transversales de flagelle, on observe des désorganisations de la disposition circulaire stricte des différents éléments de l'axonème et du périaxonème dont certains manquent.

Flagelles multiples (Figure 5)

Définition : Il y a plus d'un flagelle par spermatozoüde, la pièce intermédiaire étant commune ou multiple.

En microscopie électronique à transmission, on 
ANOMALIES DE LA PIECE INTERMEDIAIRE

\section{RESTE CYTOPLASMIQUE:}

SURFACE $>1 / 3$ TÊTE

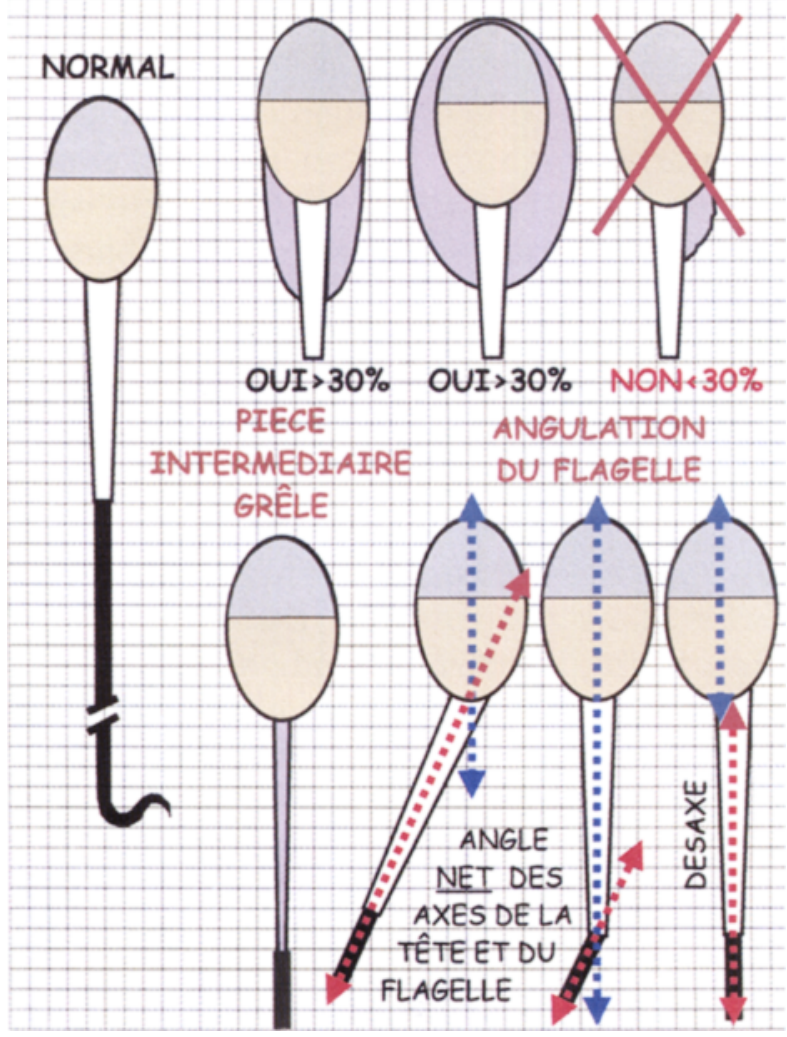

ANOMALIES DE LA PIECE PRINCIPALE

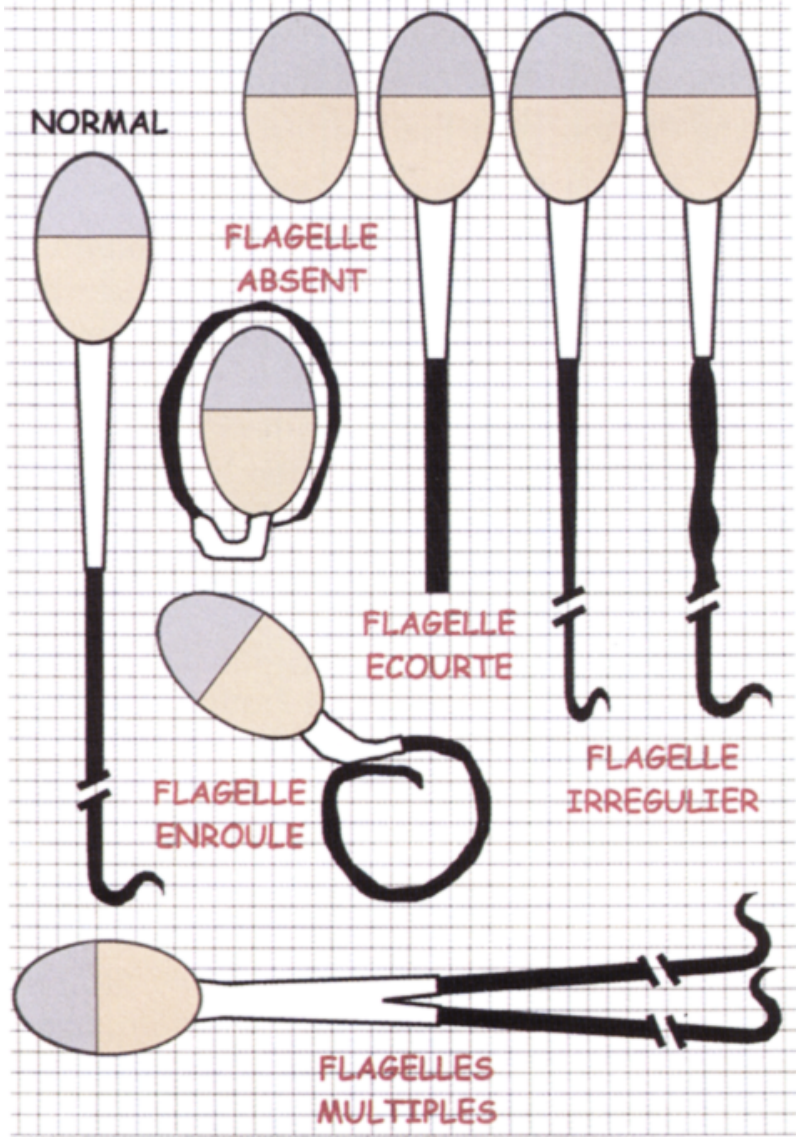

Figure 4 : Illustrations des anomalies de la pièce intermédiaire des spermatozoüdes humains.
Figure 5: Illustrations des anomalies de la pièce principale des spermatozoïdes humains. 
observe des têtes spermatiques bien isolées avec deux (et plus) plaques basales et deux (et plus) pièces connectives.

La Figure 6 donne un résumé des différentes classes d'anomalies avec les illustrations et les définitions.

\section{Exemples d'associations d'anomalies, méthode de classification et calcul de l'IAM}

Les anomalies précédemment définies sont le plus souvent associées entre elles. Aussi, comme il n'y a aucune raison objective de considérer plus une anomalie qu'une autre, on doit faire une description détaillée du spermatozoïde anormal et recenser toutes les anomalies qui sont vues en étant systématique dans l'observation et la description des anomalies pour chaque spermatozoïde observé : description de la tête puis de la pièce intermédiaire puis du flagelle.

La Figure 7 présente trois exemples de spermatozoïdes anormaux avec des anomalies associées et les catégories d'anomalies dans lesquelles ils doivent être classés. La classification et le comptage des anomalies recensées est effectué à la main ou bien à l'aide du boîtier Spermascore (Société Carteau et Ots, 93170 Bagnolet), compteur géré par microprocesseur disposé à côté du microscope et qui permet le comptage des spermatozoïdes normaux, des anomalies isolées ou associées, le calcul de l'IAM et l'impression du compte-rendu du spermocytogramme (il faut cependant noter que ce dispositif dans son état actuel limite le nombre d'anomalies recensées par spermatozoïde anormal à cinq, ce qui en toute rigueur ne permet pas une description fidèle pour des spermatozoïdes certes rares, mais qui présentent un nombre supérieur d'anomalies associées, l'observateur devant alors faire une sélection arbitraire ; un Spermascore permettant le recensement de toutes les anomalies associées possibles devrait bientôt être proposé).

Lorsque le comptage est fait à la main, on utilise une lettre minuscule pour chaque catégorie d'anomalie, c'est-à-dire de “ $a$ " pour allongé à " $p$ " pour flagelle multiple, le " $n$ " n'est pas utilisé pour qu'il n'y ait pas de confusion avec le
" $m$ ". Les associations d'anomalies sont recensées dans la grille de lecture sous forme de combinaisons de lettres. Si on reprend les 3 exemples de la Figure 7, le premier spermatozoïde présentant une tête allongée $(a)$, un acrosome anormal $(g)$, une base anormale $(f)$ et une angulation $(j)$ est répertorié $a f g j$ et cette combinaison de lettres est reportée dans chacune des cases correspondantes de la grille de lecture : dans ce cas, le spermatozoïde est donc mentionné quatre fois dans la grille (exemple du principe dans la Figure 8). Le spermatozoïde présentant une tête microcéphale $(c)$, sans acrosome $(g)$ et avec une flagelle court $(l)$ est répertorié cgl et la combinaison de lettres est reportée dans chacune des cases correspondantes. Enfin, le spermatozoïde avec une base amincie $(f)$, un reste cytoplasmique $(h)$ et un flagelle enroulé $(o)$ est répertorié fho et la combinaison de lettres est reportée dans chacune des cases correspondantes. Tout en reportant chacune des anomalies dans les différentes cases de la grille de lecture, on ne déclenche le compteur manuel ou le Spermascore qu'une fois par spermatozoïde décrit et le compte sera arrêté à 100 spermatozoïdes classés. Les spermatozoïdes normaux ou les spermatozoïdes présentant une seule anomalie sont reportés dans les catégories correspondantes à l'aide du système "bâton" qui facilite les totaux en fin d'analyse (Figure 8).

La grille de lecture présente deux colonnes dans sa partie droite destinées à effectuer les totaux (Figure 8) : la colonne de droite correspond aux totaux pour chacune des catégories d'anomalies des spermatozoïdes présentant des associations d'anomalies (combinaisons de lettres), la colonne de gauche correspond aux totaux des spermatozoïdes présentant des associations d'anomalies (c'est-à-dire le chiffre de la colonne de droite) plus les spermatozoïdes présentant uniquement l'anomalie dans la catégorie. L'étape finale est le calcul de l'IAM. On ne prend en compte pour ce calcul que le total des anomalies répertoriées dans la colonne de gauche et le total de spermatozoïdes anormaux : l'IAM est égal au rapport du nombre total d'anomalies recensées par le nombre total de spermatozoïdes anormaux. Sa plus petite valeur théorique est 1 . Un exemple de calcul est donné dans la Figure 8. 


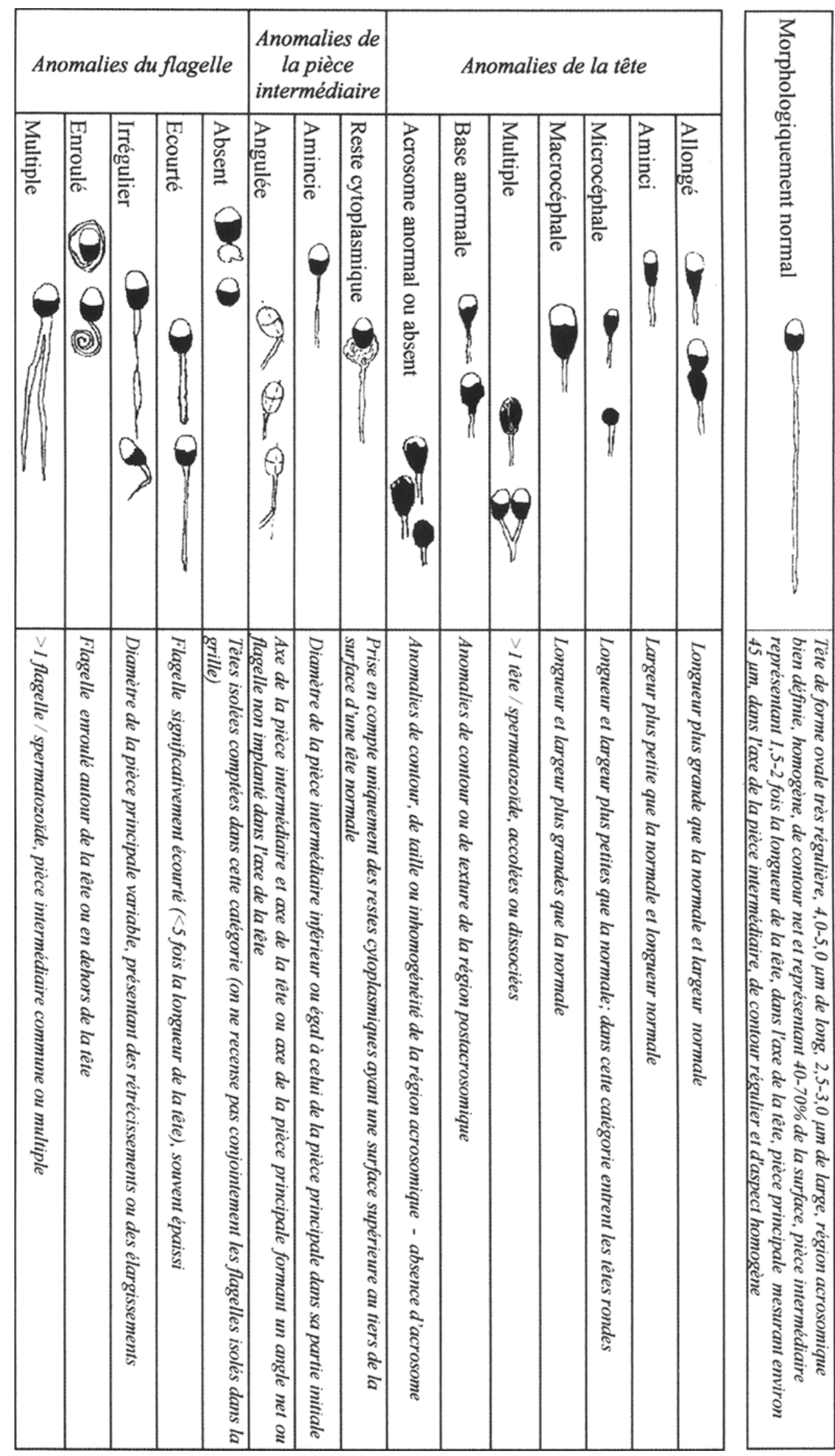

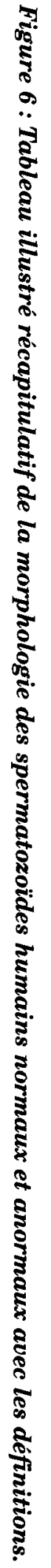




\section{SPERMATOZOÏDES AVEC DES \\ ANOMALIES ASSOCIEES}

\section{NORMAL}

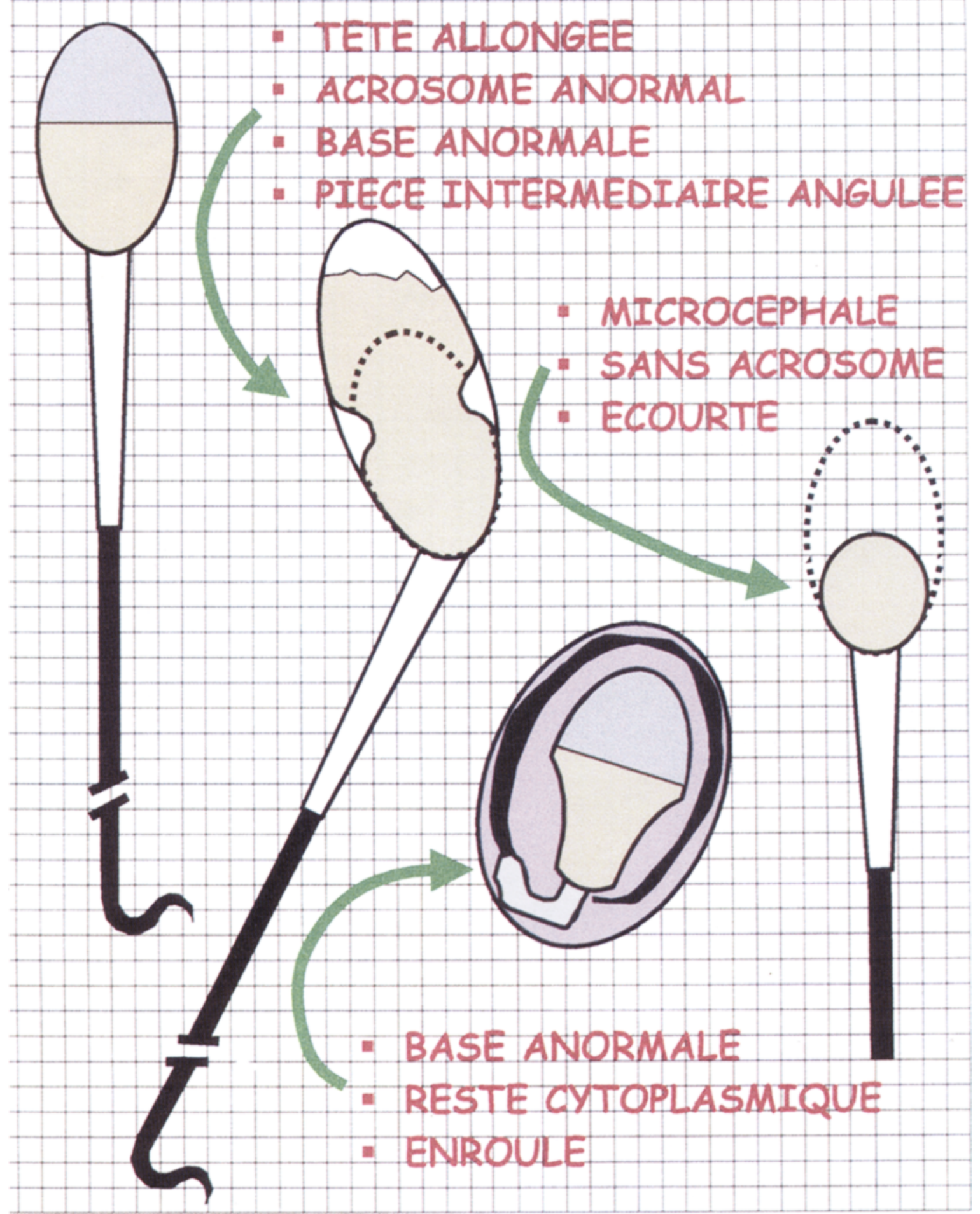

Figure 7 : Illustration et description de spermatozoüdes humains anormaux présentant des anomalies associées. 


\section{Normaux $\quad \Delta \nabla \nabla \nabla \nabla \nabla \nabla \nabla \nabla L$}

\begin{tabular}{|c|c|c|c|c|c|}
\hline \multirow{7}{*}{ 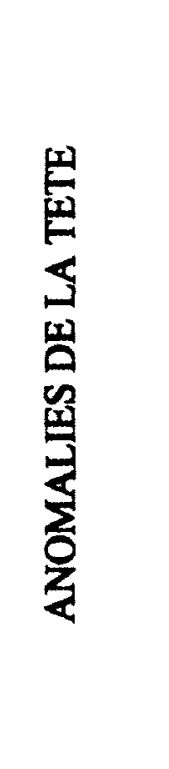 } & Allongé & $\mathbf{a}$ & & $\mathbf{0}$ & $\mathbf{0}$ \\
\hline & Aminci & b & $g b g b g b g b g b g b$ & 6 & 6 \\
\hline & Microcéphale & c & & $\mathbf{0}$ & $\mathbf{0}$ \\
\hline & Macrocéphale & d & & $\mathbf{0}$ & $\mathbf{0}$ \\
\hline & Multiple & $\mathbf{e}$ & & $\mathbf{0}$ & $\mathbf{0}$ \\
\hline & Base anormale & f & $g f g f j g f$ & 3 & 3 \\
\hline & $\begin{array}{r}\text { Acrosome anormal ou absent } \\
\square \square \square\end{array}$ & $\mathbf{g}$ & $\begin{array}{l}\text { go gi gf go go gfj gb gi gb gf go } \\
\text { gb gb go ghj gl gb gb go go gh } \\
\text { gh gh go gh }\end{array}$ & 39 & 25 \\
\hline \multirow{3}{*}{ 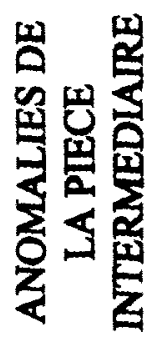 } & Reste cytoplasmique & $\mathbf{h}$ & ghj gh gh gh gh & 8 & 5 \\
\hline & Amincie & $\mathbf{i}$ & & $\mathbf{0}$ & $\mathbf{0}$ \\
\hline & Angulée & $\mathbf{j}$ & $g j g f j g j g h j$ & 7 & 4 \\
\hline \multirow{5}{*}{ 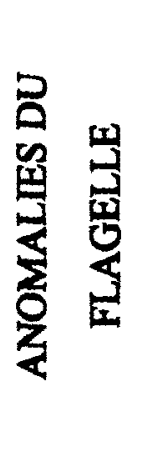 } & Absent & $\mathbf{k}$ & & $\mathbf{0}$ & $\mathbf{0}$ \\
\hline & Court & 1 & $g l$ & 1 & 1 \\
\hline & Irrégulier & $\mathbf{m}$ & & $\mathbf{0}$ & $\mathbf{0}$ \\
\hline & Enroulé & o & go go go go go go go go & 16 & 8 \\
\hline & Multiple & $\mathbf{p}$ & & $\mathbf{0}$ & $\mathbf{0}$ \\
\hline
\end{tabular}

\begin{tabular}{|l|c|}
\hline Nombre total d'anomalies $=T$ & 80 \\
\hline IAM $=$ T / Nb de spermatozoides anormaux $:$ & 1,51 \\
\hline
\end{tabular}

Figure 8 : Classification par la méthode de David modifiée: exemple de grille de lecture remplie et calcul de l'IAM. 


\section{CONCLUSIONS}

La méthode de David modifiée présente un grand intérêt pour le diagnostic et le pronostic dans la prise en charge des couples inféconds car elle permet la prise en compte des associations d'anomalies morphologiques à côté de l'évaluation de la fréquence de spermatozoïdes normaux. Cependant, la classification des spermatozoïdes anormaux qui semble $a$ priori être une tâche simple est dépendante de nombreux facteurs de variation. Aussi, ce n'est qu'au prix d'une standardisation de l'analyse incluant, au premier chef, l'étape analytique, mais aussi toutes les autres étapes, de la préparation des lames au rendu des résultats, d'un entraînement régulier et de la pratique de contrôles de qualité, que les plus faibles variabilités intra-, inter-observateur et inter-laboratoire pourront être obtenues. Cette optimisation de l'analyse est un pré-requis indispensable pour atteindre une meilleure fiabilité des analyses tant d'un point de vue clinique que de recherche.

\section{RÉFÉRENCES}

1. AUGER J., SCHOEVAERT D., NEGULESCO I., DADOUNE J.P. : The nuclear status of human sperm cells by TEM image cytometry: Relation of nuclear shape and chromatin texture to fertility. J. Androl., 1993, $14: 456-463$.

2. BISSON J.P., DAVID G. : Anomalies morphologiques du spermatozoïde humain. 2) Etude ultrastructurale. J. Gynecol. Obstet. Biol. Reprod., 1975, (Suppl.1) : 3786.

3. BONDE J.P., ERNST E., JENSEN T.K. et al. : Relation between semen quality and fertility: a population-based study of 430 first-pregnancy planners. Lancet, 1998, 352 : 1172-1177.

4. BOYERS S.P., DAVIS R.O., KATZ D.F. : Automated semen analysis. Curr. Probl. Obstet. Gynecol. Fertil., $1989,12: 165-200$.

5. ClaVERT A., BOURGUIGNAT A., SIEST J.P., FERARD G. : Enquête sur les pratiques du spermogramme en France. Andrologie, 1997, 7 : 443-449.

6. DAVID G., BISSON J.P., CZYGLIK F. et al. : Anomalies morphologiques du spermatozoïde humain. 1) Propositions pour un système de classification. J. Gynecol. Obstet. Biol. Reprod., 1975, (Suppl.1) : 1736.

7. EGGERT-KRUSE W., SCHWARZ H., ROHR G. et al. : Sperm morphology assessment using strict criteria and male fertility under in-vivo conditions of conception. Hum. Reprod., 1996, 11 : 139-146.
8. ESHRE ANDROLOGY SPECIAL INTEREST GROUP : Guidelines on the application of CASA technology in the analysis of spermatozoa. Hum. Reprod., 1998, 13 : 142-145.

9. FENSTER L., KATZ D.F., WYROBEK A.J. et al. : Effects of psychological stress on human semen quality. J. Androl., 1997, $18:$ 194-202.

10. JEULIN, C., FENEUX, D., SERRES, C. et al. : Sperm factors related to failure of human in vitro fertilization. J. Reprod. Fertil., 1986, 76 : 735-744.

11. JOUANNET P., DUCOT B., FENEUX D., SPIRA A. : Male factors and the likelihood of pregnancy in infertile couples. I. Study of sperm characteristics. Int. J. Androl., 1988, $11: 379-384$.

12. KRUGER T.F., ACOSTA A.A., SIMMONS K.F. et al. : Predictive value of abnormal sperm morphology in in vitro fertilization. Fertil. Steril., 1988, 49 : 112-117.

13. LIM C.C., LEWIS S.E., KENNEDY M., DONNELLY E.T., THOMPSON W. : Human sperm morphology and in vitro fertilization: sperm tail defects are prognostic for fertilization failure. Andrologia, 1998, 30 : 43-47.

14. MAC LEOD J. : Effects of environmental factors and of antispermatogenic compounds on the human testis as reflected in seminal cytology. In : Mancini R.E, Martini L. eds. Male Fertility and Sterility. New York, Academic Press, 1974 : 123-148.

15. MORRUZZI J.F., WYROBEK A.J., MAYALL B.H., GLEDHILL B.L. : Quantification of human sperm morphology by computer-assisted image analysis. Fertil. Steril., 1988, $50:$ 142-152.

16. ORGANISATION MONDIALE DE LA SANTE : Manuel de Laboratoire de l'OMS pour l'Analyse du Sperme Humain et de l'Interaction des Spermatozoïdes avec le Mucus Cervical. Traduction française (Auger J., Jouannet P.) de : WHO laboratory manual for the examination of human semen and sperm-cervical mucus interaction (3rd ed). Editions INSERM, Paris, 1993.

17. STEENO O.P., PANGKAHILA A. : Occupational influences on male fertility and sexuality. Andrologia, 1984, $16: 5$-22.

18. TONER J.P., MOSSAD H., GROW D.R. et al. : Value of sperm morphology assessed by strict criteria for prediction of the outcome of artificial [intrauterine] insemination. Andrologia, 1995, 27 : 143-148.

19. WORLD HEALTH ORGANIZATION : WHO laboratory manual for the examination of human semen and sperm-cervical mucus interaction. 3rd ed. Cambridge, Cambridge University Press, 1992.

20. WYROBEK, A.J., GORDON, L.A., BURKHART, J.G. et al. : An evaluation of human sperm as indicators of chemically induced alterations of spermatogenesis in man. Mutation Res., 1983, 115 : 73-148. 


\section{ABSTRACT}

Standardization of the morphological assessment of human spermatozoa according to modified David's classification

\section{J. AUGER, F. EUSTACHE}

It has now been clearly established that the percentage of morphologically normal sperm and the level of several specific anomalies of spermatozoa have a prognostic value in vivo and in vitro. Assessment of human sperm morphology therefore represents an essential step in routine semen analysis. Smears, preferably stained by the Shorr technique, are examined at a final magnification of $\times 1000$ ( $\times 100$ objective with oil immersion and x10 ocular lens). David's method for classification of morphological anomalies of human spermatozoa was proposed in the mid-seventies and was modified in the early nineties to account for all of the defects known to interfere with normal sperm functions. This method has been adopted in France by the vast majority of public and private laboratories. It allows classification of seven anomalies of the head: tapered, thin, microcephalous, macrocephalous, multiple, abnormal or absent acrosome, abnormal postacrosome, three anomalies of the midpiece: cytoplasmic droplet, thin, bent, and five anomalies of the principal piece: absent, short, irregular, coiled and multiple. The originality of the method is to account for all combinations of anomalies found for each abnormal sperm by means of a multiple entry system. Since there is no objective reason to favour certain anomalies over others, all anomalies found for each abnormal sperm should be recorded using a systematic procedure: description of the head, followed by the midpiece, and lastly the principal piece. The multiple anomalies index or MAI is calculated. This index, which is a direct application of the multiple entry system, corresponds to the mean number of anomalies per abnormal sperm (the ratio of the total number of anomalies to the number of abnormal sperm). Several studies have indicated the prognostic value of MAI, and calculation of the MAI is recommended in the WHO manual on semen analysis. Unfortunately, the morphological assessment of human spermatozoa which appears simple at a first glance, raises a number of real difficulties: this microscopic analysis is highly subjective by nature and no uniform recommendations for staining technique and classification have yet been defined. Internal and external quality control schemes have revealed the marked intra- and interindividual variability of the results, which in turn have important consequences on the reliability of the analysis and the management of infertile couples. All steps of the procedure, from preparing the smear to writing the examination report must therefore be standardised. The present article focuses on standardisation of the classification step using David's method. Precise descriptions of the anomalies found by conventional microscopy and their ultrastructural substratum are presented, together with practical recommendations for the classification of sperm defects and calculation of the MAI.

Key words: Sperm morphology assessment, human spermatozoa, David's method, multiple anomalies index, standardisation, quality insurance 